\title{
Analysis of traffic corridor impacts from the introduction of the new Athens Metro system
}

\author{
J.C. Golias * \\ Department of Transportation Planning and Engineering, Faculty of Civil Engineering, National Technical University of Athens, \\ ZOGRAFOU Campus, 5 Iroon Polytechniou Street, Athens 157 73, Greece
}

\begin{abstract}
New transit systems are viewed as an effective approach to dealing with concerns about automobile dependence and a degrading quality of life in many large cities. This paper concentrates on evaluating the impacts from the construction of a new subway system as these impacts pertain to traveler behavior and mode choice. Using the results from a revealed preference combined roadside and on-board survey from Athens, Greece, and utilizing a flexible disaggregate model specification (the heteroskedastic extreme value model) the results from the introduction of a new Metro system are evaluated. The results indicate that the demand for auto usage is fairly inelastic (with respect to both cost and time), and that Athens transit users are more sensitive to changes in cost rather than travel time. Further, the results indicate that increases in travel time and cost for the auto would increase the demand for the Metro, but not as much for the bus. (c) 2002 Elsevier Science Ltd. All rights reserved.
\end{abstract}

Keywords: Metro system; Traffic impacts; Mode choice

\section{Introduction}

Traffic congestion, automobile dependence, car ownership, emissions pollution, and a decreasing quality of life are often cited as some of the most important problems faced by today's large urban centers. In an effort to address some of these problems, many cities have invested sizeable amounts in the extension and redevelopment of old and the creation of new urban public transport systems. It is hoped that these public transport systems will help combat the increased dependence on automobile and the associated problems. Among public transport systems, it has been argued that rail-based systems can play a critical role in overcoming the problems posed by the private automobile (Vuchie, 1991; Newman, 1995; Thornblom and Bengtsson, 1997; Ahern, 2001).

Obviously, most of the arguments in favor of new public transportation systems are based on the perceived impacts: increased market share for public transportation, reduced automobile dependence, environmental impacts, and effects on urban development. Much of the effort for developing new systems has concentrated,

\footnotetext{
Tel.: +30-1-772-1276; fax: +30-1-772-1327

E-mail address: igolias@central.ntua.gr (J.C. Golias).
}

worldwide, in the direction of Metros (or subways). These developments offer unparalleled quality of service (in terms of frequency and speed of service and reduced travel time) and can, with greater probability than purely bus-based systems, act as a deterrent to automobile use. Many authors have examined the impacts of new public transport systems around the world. Mackett and Edwards (1998) examined, with the use of extensive questionnaires, the expected and realized benefits from the construction of new public transport systems from 100 cities around the world. Their results indicate that the impacts of the new urban public transport systems are much smaller than those anticipated by promoting them (the authors of this study also offer an extensive review of much of the literature on the expected and realized benefits from the construction of new public transport systems). Harper (2000) examined the impacts of the opening of lines 1 and 2 of the Midland Metro (West Midlands region of England) with the use of a household survey. One of Harper's most interesting results was that transfer from car makes up a smaller proportion of patronage than forecast. Monzon (2000) investigated the travel demand impacts of a new privately operated subway system in Madrid. The author indicated that $75 \%$ of the travelers had been transferred from other modes $(65 \%$ from bus and $35 \%$ from private automobile), while $25 \%$ was generated traffic, due to 
both new housing developments and changes in work destinations. In contrast, Manchester's Metrolink secured a much larger modal shift from private car to light rail than forecast (Knowles, 1996).

While these studies offer significant insights into the effects of new public transport systems especially as they compare to anticipated changes, they have been primarily concerned with financial and ridership figures. This paper concentrates on evaluating the impacts from the construction of a new subway system as these pertain to traveler behavior and mode choice. Examining mode choice decisions of travelers on the traffic corridors served by the new subway system in Athens,
Greece, results can be drawn regarding the user characteristics of the various modes, the elasticities and cross-elasticities of different mode use, and the decision process of the travelers. The remainder of this paper is organized as follows. Section 2 examines some of the characteristics of the Athens urban transport system, offers some figures on the new subway system development and presents some of the initial impacts. Section 3 outlines the mode choice model formulation used in this paper and overviews the data used to calibrate the model. Section 4 presents the estimation results and Section 5 discusses the findings of this paper and offers some concluding remarks.

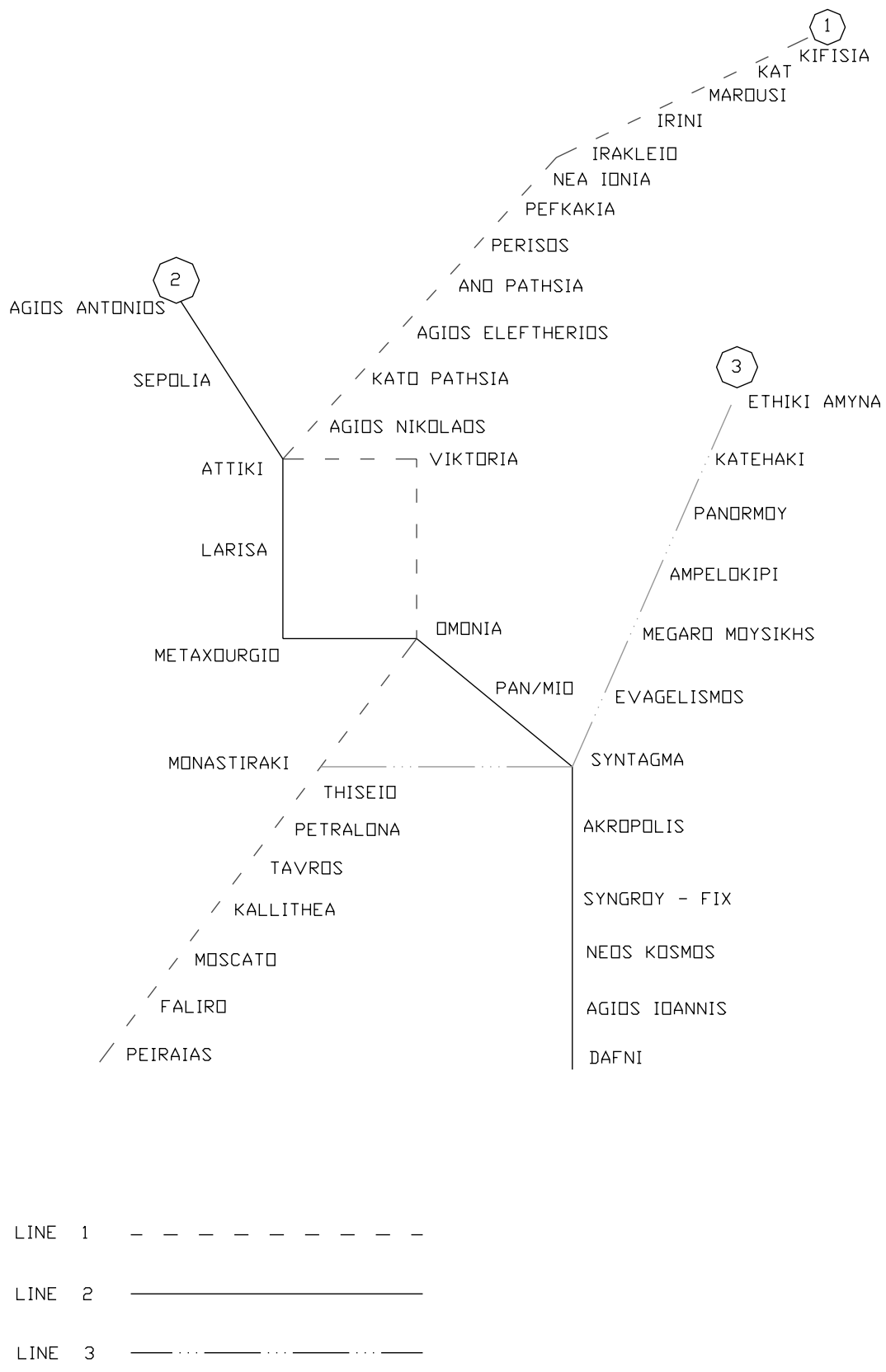

Fig. 1. The map of the Athens Metro. 


\section{Background}

\subsection{The Athens, Greece, urban transport system}

The urban area of Athens, the capital of Greece, has an area of $1470 \mathrm{~km}^{2}$ and a population of approximately 3.7 million people. During the last decade the population in the greater Athens area has increased by about $10 \%$ while at the same time car ownership has increased considerably, approaching 250 private automobiles per 1000 inhabitants in 1996 (car ownership in 1983 was 172 automobiles per 1000 inhabitants). This has led to an increase in travel time by $26 \%$ in the last 12 years which, along with the insufficient urban road network in the central areas, has led to a deterioration of traffic conditions in the capital.

For the Athens Metropolitan area, there is a daily demand for 5,650,000 journeys, with a 1,080,000 2-h peak demand. There are 6,300,000 single mode daily trips, a $26 \%$ increase in the last 12 years. From this information, it appears that there are 1.72 daily trips per person (1.54 journeys/person), while $55 \%$ of the population makes at least one trip per day, with business and education trips $(45 \%$ and $15 \%$ of total daily travel, respectively) having the highest share (Metro Development Study, 1996b).

In principle, the public transport planning process for the greater Athens Metropolitan area is done by OASA, the Athens Urban Transportation Organization. According to OASA's data and reports, Athens is served by a mass transit system of 1840 buses, 1500 of which are in operation daily, 356 trolley buses, 290 of which are in operation daily, and 3 Metro lines with 269 cars. The bus system is made up of 41 trunk lines, 116 central lines, 9 intermunicipal lines, 98 local-feeder lines, eight express lines, and 6 school lines, with a total annual ridership of 403 million passengers. This ridership is complemented by 90 million annual ridership for the trolley buses, and 92 million passengers for Metro. Transit providers have to serve a system which faces a $3.5 \%$ annual increase in traffic for the last 10 years and which has $22 \%$ of the signalized intersection approaches in the center of the city highly congested (Metro Development Study, 1996a). Obviously, the provision of high-level transit services in such a congested network is very difficult.

\subsection{The new Athens Metro system}

From the previous discussion it is apparent that Athens, similar to many other of the World's larger cities, is suffering from traffic congestion and related problems such as a diminished quality of life, environmental degradation, etc. As a means toward dealing with many of these problems, a decision was made to build a new subway system to act as a complement to the existing urban rail line and the urban transport system.

The new Metro system (two new lines), together with an existing $25 \mathrm{~km}$ long line, makes up the body of a new, mode complete, fast and effective urban transport network (Fig. 1). The decrease in private car trips, as well as the restricted use of city buses to and from the center, are hoped to eventually contribute to the improvement of traffic, but also to a cleaner atmosphere over the greater Athens region, as many bus lines end now at Metro stations outside the center. One of the goals of the construction was to encourage the public by significantly increasing their use of public transportation services, in particular the expanded Metro system and coordinated surface lines, as the preferred alternative to driving their automobiles through the streets of a congested city (information on Metro and bus line coordination plans and maps can be found at http:// www.oasa.gr/sas.htm). This is expected to bring substantial environmental benefits to the community as a whole, such as helping to reduce Athens' severe air pollution and ambient noise level problems.

The new underground Metro system consists of two lines radiating in four directions from the center of the city, with a total of 21 stations and a capacity to serve approximately 450,000 daily passengers (This is in addition to the 330,000 passengers currently being served by the existing Metro line). Trains run every $3 \mathrm{~min}$ in rush hour, and every 5-10 min in non-rush hour. The total cost was approximately 2.3 billion ECU in 1999 currency values. The European Union (EU) and the European Investment Bank (EIB) financed up to $90 \%$ of the project through loans and grants, while the remaining funds were provided by the Greek government.

\subsection{Data collection}

The data needed to assess behavior as well as changes in behavior by the presence of a new mode of transport come from questionnaires. The survey used in this paper included a large number of inquiries, including questions on mode choice, travel time, travel cost, trip purpose, socioeconomic characteristics, and trip chain behavior. The surveys were completed in two locations: First, on board, with face to face interviews for Metro and bus riders, and second, with roadside interviews for automobile users. It should be mentioned here that the main goal of this survey was to assess both the underlying characteristics of mode choice and the changes in mode choice behavior from the introduction of the new Metro system. In order to select the most representative sample of bus and Metro riders, a multi-stage stratified sampling process was followed.

The strata of the survey were the two main modes considered, buses and Metro, and the six different types of lines within the bus network. From each stratum, a 
random sample of lines was selected, the size of which was proportional to the ridership of the stratum, with the probability of selecting each line proportional to its ridership (proportional to size sampling). Finally, weighted random sampling (using age and sex as the weights) was used to select the interviewed individuals.

To avoid the problem of non-response from automobile users, help from the police was requested. A police officer was present during the entire duration of the roadside interviews, to stop drivers and ensure that they would respond to the questionnaire. For the on board surveys, the response rate was very high (over $90 \%$ ), since an explicit effort was made to interview passengers who just boarded the bus or Metro and had adequate time ahead of them before getting off the bus or Metro. A total of 4200 complete questionnaires were collected, larger than most other mode choice studies. The sample collected suggests that $71 \%$ of the riders use transit on a daily basis and $24 \%$ uses the system 1-3 times a week. It can be seen that the sample of this study, and more generally the public using the system, is made up of frequent users as $95 \%$ of the individuals surveyed use transit at least weekly.

\subsection{Initial impacts of interest}

The initial result that is of interest deals with the mode most commonly used by Metro riders before the system's opening. As can be seen from Table 1, more than half of Metro's current ridership has been diverted from other modes of public transportation (53\% from the buses and $3 \%$ from the train), while $24 \%$ has been attracted from private modes (16\% from auto, $1 \%$ moto and $7 \%$ from taxi). Interestingly, $3 \%$ of the trips were walking trips (this is particularly true for trips within the CBD), and $2 \%$ of the travel was induced.

The results of the survey uncovered some interesting characteristics for commuters in the traffic corridors served by the new Metro system. It appears that a large percentage of bus and Metro riders use a combination of modes to get to their destination (Table 2). For example, about half $(51 \%)$ of the bus riders use only the bus for their trip while $43 \%$ uses a combination of two modes (bus-Metro or bus-auto or bus-taxi) and 6\% uses three

Table 1

Mode(s) used by Metro riders before new system opening

\begin{tabular}{lr}
\hline Previous mode & \\
\hline Bus & $53 \%$ \\
Train & $3 \%$ \\
Private (auto, moto, taxi) & $24 \%$ \\
Bus/auto & $5 \%$ \\
Bus/train & $7 \%$ \\
Train/auto & $2 \%$ \\
Walk & $3 \%$ \\
Induced trip & $2 \%$ \\
\hline
\end{tabular}

Table 2

Number of modes used

\begin{tabular}{llll}
\hline & 1 & 2 & 3 \\
\hline Bus riders & $51 \%$ & $43 \%$ & $6 \%$ \\
Metro riders & $26 \%$ & $57 \%$ & $17 \%$ \\
\hline
\end{tabular}

modes. The situation in the Metro system is quite similar. Partly due to the limited network coverage offered by the new Metro system, $57 \%$ of Metro's riders use another mode to complete their journey. As a result, it can be inferred that combining modes to complete trips in the traffic corridors served by the new Metro system is commonplace.

\section{Mode choice model formulation}

As is well known, the topic of commuter mode choice has attracted considerable attention in the scientific literature, as a consequence of the useful and practical conclusions that can be drawn from such an analysis. A review of the literature, both in terms of the methodologies and the findings are beyond the scope of this paper; nevertheless, it is mentioned that Hensher and Ton (2000) and De Palma and Rochat (2000) offer a thorough overview of the methodologies and case study findings for the various mode choice approaches used.

The most widely used models for mode choice are of disaggregate nature, and use questionnaire data to be estimated. The models that describe traveler behavior are based on the utility maximization principle, under time and cost constraints. This utility, which an individual associates with an alternative, is specified to be the sum of a deterministic component (which is a function of the observed attributes) and the random component (capturing the unobserved characteristics of the alternative). Early mode choice work was done under the assumption of independent and identically distributed (IID) random components across the utilities, yielding the widely used multinomial logit model (MNL). MNL models have a closed-form mathematical structure which makes it fairly straightforward to estimate, but are hindered by the highly unrealistic independence from irrelevant alternatives property (IIA) which imposes the restriction of equal cross-elasticities due to a change in an attribute affecting only the utility of a given alternative.

Removing the IID assumption of the MNL model can result in overcoming the rigid IIA property imposed on the alternatives. One such model structure is the hierarchical nested logit (NL) model which overcomes the IIA property. This flexibility of the NL models has made it an extremely popular choice for modeling mode choice. The NL model would be a good choice for modeling the mode choice behavior of commuters in 
Athens, but, as it could be seen from Table 2, Athens' system is based on modal transfers. That is, almost half of the commuters that use the bus or the Metro, will complete their trip using another mode as well. And, this is where a relative weakness of the NL model lies. It is fairly onerous and complicated to model mode combinations when using the NL model.

To overcome this difficulty, there are two categories of models that both remove the IIA property and can accommodate the fairly complex behavior of combining modes. The first is the multinomial probit model (MNP) and the second is the hierarchical extreme value logit model (HEV) (Bhat, 1995). Because, as has been widely reported in the literature, the estimation of the MNP model generates a number of conceptual, statistical and practical problems, including difficulty in interpretation, highly non-intuitive model behavior and low-parameter estimates precision (Bhat, 1995), it was decided to use the HEV model in this paper. It should be noted here that only recently has the HEV model gained wider acceptance; this is because only recently have a few software packages included this specification in the standard gamut of discrete choice models. In this paper only the essential parts of the HEV model are presented. Readers interested in the original analysis should refer to Bhat (1995) and Washington et al. (2002) give an in-depth view on the development, estimation, transferability, and comparative advantages of the various discrete model specifications. The HEV model is based on the same principles as the MNL model. As such, it is considered that the selection of mode is a function of a number of independent variables $X_{i}$ and of the random utility $\varepsilon_{\mathrm{i}}$. Thus, the utility function can be written as follows:

$U_{i}=V_{i}\left(X_{i}\right)+\varepsilon_{i}$.

But, instead of assuming that the terms $\left(\varepsilon_{i}\right)$ are IID based on the inverse exponential distribution as is the case for the MNL model, in the HEV model the hypothesis is made that these terms are independently but non-identically distributed based on the type I extreme value distribution. From the extreme value distribution it follows that probability density function and the cumulative distribution function of the terms $e_{i}$ are given by

$f\left(\varepsilon_{i}\right)=\frac{1}{\theta_{i}} \mathrm{e}^{-\left(\varepsilon_{i} / \theta_{i}\right)} \mathrm{e}^{-\mathrm{e}^{-\left(\varepsilon_{i} / \theta_{i}\right)}}$,

$F_{i}(z)=\int_{\varepsilon_{i}=-\infty}^{\varepsilon_{i}=z} f\left(\varepsilon_{i}\right) \mathrm{d} \varepsilon_{i}=\mathrm{e}^{-\mathrm{e}^{-\left(z / \theta_{i}\right)}}$.

The $\varepsilon_{i}$ terms have variance equal to $\theta_{i}$. By combining Eqs. (1) and (3), the probability $P(i)$ that a passenger will select mode $i$ from the set of available modes $(C)$ is

$$
\begin{aligned}
P(i) & =\operatorname{Prob}\left(U_{i}>U_{j}\right) \quad \forall j \neq i, j \in C \\
& =\operatorname{Prob}\left(\varepsilon_{j} \leqslant V_{i}-V_{j}+\varepsilon_{i}\right) \quad \forall j \neq i, j \in C \\
& =\int_{\varepsilon_{i}=-\infty}^{\varepsilon_{i}=+\infty} \prod_{j \in C, j \neq 1} \Lambda\left[\frac{V_{i}-V_{j}+\varepsilon_{i}}{\theta_{j}}\right] \frac{1}{\theta_{i}} \lambda\left(\frac{\varepsilon_{i}}{\theta_{i}}\right) \mathrm{d} \varepsilon_{i},
\end{aligned}
$$

where $\lambda(\cdot)$ and $\Lambda(\cdot)$ are the pdf and cdf of the type I extreme value distribution. The parameters of the HEV model can be straightforwardly estimated using maximum likelihood estimation. In this analysis, a passenger has five alternative modes to select from: bus only, bus and Metro, Metro only, Metro and auto, auto only. It is also worth noting that for each passenger, besides mode choice information, data on mode and socio-economic characteristics were collected.

\section{Estimation results}

Using the data previously described and the HEV model specification, this section analyzes the model results and some of the most interesting findings yielded by this analysis. It should be noted that a number of different variable specifications were examined to determine the preferred utility function specification. The final specification was reached by eliminating the variables found to be non-significant or completely counterintuitive in previous specifications. Among others, variables such as education, household size, number of adults, number of available automobiles in the household were tested. As expected, it was found that both travel costs and travel time play an important role in mode choice (Table 3). Overall, the signs of the

Table 3

Mode choice estimation results

\begin{tabular}{lcc}
\hline & Parameter & $t$-Statistics \\
\hline Travel cost & -0.0415 & -18.9 \\
Travel time & -0.0034 & -9.4 \\
Mode constants (auto is base) & & \\
Bus & -0.2 & -10.2 \\
Bus/Metro & -0.31 & -2.96 \\
Metro & -0.21 & -2.85 \\
Metro/auto & -0.3 & -2.58 \\
Rider income (auto is base) & & \\
Bus & -0.085 & -3.98 \\
Bus/Metro & -0.0088 & -4.48 \\
Metro & -0.001 & -1.01 \\
Metro/auto & 0.0003 & 0.77 \\
Rider age (auto is base) & & \\
Bus & -0.11 & -5.59 \\
Bus/Metro & -0.034 & -3.79 \\
Metro & 0.13 & 3.05 \\
Metro/auto & 0.01 & 1.06 \\
No. of questionnaires & 4481 & \\
Likelihood ratio index & 0.32 & \\
\hline
\end{tabular}


Table 4

Value-of-time for Athens commuters (values in Euros)

\begin{tabular}{|c|c|c|c|c|c|c|}
\hline & All & Bus & Bus/Metro & Metro & Metro/auto & Auto \\
\hline Total & 2.16 & 1.60 & 1.82 & 2.49 & 2.82 & 2.93 \\
\hline \multicolumn{7}{|l|}{ Income } \\
\hline High & 2.64 & 1.97 & 2.24 & 3.14 & 3.57 & 3.97 \\
\hline Medium & 2.20 & 1.56 & 1.90 & 2.61 & 2.93 & 3.35 \\
\hline Low & 1.76 & 1.32 & 1.50 & 2.03 & 2.35 & 2.46 \\
\hline \multicolumn{7}{|l|}{ Purpose } \\
\hline Work & 2.25 & 1.67 & 1.98 & 2.64 & 3.33 & 3.64 \\
\hline Shopping & 2.79 & 2.07 & 2.40 & 3.31 & 3.94 & 4.03 \\
\hline
\end{tabular}

parameters were consistent with expectations and the choice of the automobile was taken as the base for comparisons. There is a preference for the automobile, ceteris paribus, higher income shows a clear preference for the automobile over other modes of travel, while older people prefer the automobile over the bus (this is not the case for the Metro, where age increases lead to a higher utility for the Metro than the automobile). It should be mentioned that income was not considered as a continuous variable, since many people refuse to answer regarding their exact income, but as a grouping variable with three classes: low, medium and high income. Age was given again as a discrete variable with six groupings. Finally, the statistical fit of the model was evaluated using the likelihood ratio index (Washington et al., 2002), which is a measure similar to $R^{2}$ for linear regression; values in the $0.25-0.50$ range for a non-linear fit are considered as quite good.

Of course, despite the interest in the sign and magnitude of the parameters themselves, most of the interest in such analyses shifts from the parameters to findings of value-of-time (VoT) and of elasticities. Table 4 presents the VoT findings for the entire sample, for each travel mode, and the variations by income and purpose of travel (all values are in Euros). The VoT for the total sample was found to be 2.16 Euros. This value varies between 1.60 Euros for travel by bus only and 2.93 Euros for travel by auto only. It is of interest to note that the VoT for Metro, Metro/auto and auto is similar, with Metro's VoT being much closer to that of the auto than to that of the bus, as it may have been expected. Also as expected is the finding that increases in income lead to increased VoT, regardless of mode, and when shopping is the purpose of travel the VoT is much higher than when work is the purpose.

Another very important finding is that of the elasticities of each mode with respect to changes in its travel cost and travel time (Table 5). It appears that increases in travel cost will have a higher impact on bus travel rather than auto travel. For car users, elasticities with respect to travel time and cost are -0.33 and -0.27 , respectively; for bus users these elasticities are -0.44 and -0.58 , and finally for Metro users they are -0.31 and
-0.33 . These results show that the demand for auto as well as for Metro usage is fairly inelastic (with respect to both cost and time), and that the users of both modes present a similar behavior with respect to cost and time changes. Furthermore, the mode choice elasticities reveal that Athens transit users are more sensitive to changes in cost rather than travel time.

In contrast to the own elasticities, which show the changes in demand from a change in one of the characteristics of a mode, cross-elasticities examine the changes in a mode's demand by changes in the characteristics of other modes (Table 6). Increasing bus cost would lead to an increase in the demand for the Metro and the auto. More importantly though, an increase in travel time would lead to increases which are higher than those due to cost increases for the auto but lower for the Metro. These results seem to confirm the above findings that transit users are more sensitive to changes in cost than changes in time. Furthermore, they seem to indicate that auto users present the lower sensitivity to cost changes and time changes although the latter is not

Table 5

Mode choice elasticities

\begin{tabular}{lll}
\hline & Cost & Time \\
\hline Bus & -0.58 & -0.44 \\
Bus/Metro & -0.41 & -0.37 \\
Metro & -0.33 & -0.31 \\
Metro/auto & -0.31 & -0.37 \\
Auto & -0.27 & -0.33 \\
\hline
\end{tabular}

Table 6

Mode choice cross-elasticities for selected modes

\begin{tabular}{llll}
\hline & \multicolumn{2}{l}{ Elasticities } & \\
\cline { 2 - 4 } & Bus & Metro & Auto \\
\hline Bus changes & & & \\
Cost & -0.58 & 0.53 & 0.21 \\
Time & -0.44 & 0.41 & 0.31 \\
Auto changes & & & \\
Cost & 0.27 & 0.52 & -0.27 \\
Time & 0.37 & 0.87 & -0.33 \\
\hline
\end{tabular}


considerably different than that for Metro users. When it comes to travel time and travel cost increases for the auto it is worth mentioning that demand increase is significantly lower for the bus than it is for the Metro. This seems to indicate strongly that the users' attitude towards these two modes is considerably different and as a result attraction of riders from the private car market share is easier for the Metro than it is for the buses.

\section{Discussion and conclusions}

This paper concentrated on examining the results on mode choice from the introduction of a new Metro system in the city of Athens, Greece. In the first part of the paper these results are viewed from a simple descriptive point of view, where it appears that the new Metro system has attracted a large number of bus riders $(53 \%)$ and a smaller number of private car users $(24 \%)$. The analysis also suggests that contrary to what Monzon (2000) reported, generated traffic for the Athens Metro was quite low (2\%). Nevertheless, this new Metro line, with a 250,000 passenger daily ridership, has helped alleviate a part of the traffic problem, at least for the traffic corridor it serves.

The second part of the paper concentrated on estimating a random utility model which does not violate the IIA property of the MNL model, yet is flexible enough to overcome some of the methodological constraints of the NL model. The HEV model estimates allowed for interesting insights into the mode choice decisions of travelers in Athens. Overall, it seems that bus riders are more sensitive to both changes in travel time and cost than any other mode. On the contrary, the passenger car seems to present the lowest sensitivity with respect to these two parameters, especially travel cost. This is a rather interesting result from a policy perspective, as the very low elasticity of auto travel may raise significant questions regarding the effectiveness of monetary measures aimed at controlling private car usage. Furthermore, it is worth noting that travel time seems to have a comparative impact on Metro and auto users. This result indicates that Metro - at least for this case study - is considered as a public transport mode which may compete operationally with the passenger car.

Thus, the introduction of a Metro system is expected to upgrade considerably public transport within the influence area of the travel corridor it serves. The size of this positive influence depends of course on a number of parallel policies adopted for the support of Metro operations, e.g. implementation of park-n-ride initiatives in the context of an integrated parking policy. Moreover, it should be mentioned that any attempt to increase the public transport market share may also require actions towards discouraging the use of private cars which, taking into account the low-cost elasticity of auto usage, may range from measures increasing the private car travel time to complete banning of private cars along a number of lanes of a corridor or along the whole corridor or within a specific area. A number of free lanes may be used for upgrading the quality of pedestrian movements while the remaining may be used exclusively by buses in an effort to improve bus travel time, and thus increase significantly bus ridership, as the sensitivity analysis of this mode indicates.

\section{References}

Ahern, A., 2001. Model choices and new urban public transport. Traffic Eng. Control 42 (4), 108-114.

Bhat, C.R., 1995. A heteroskedastic extreme value model of intercity travel mode choice. Transp. Res. B 29, 471-483.

De Palma, A., Rochat, D., 2000. Mode choice for trips to work in Geneva: an empirical analysis. J. Transp. Geography 8, 43-51.

Harper, R., 2000. Midland metro: monitoring the impacts, Presented in the European Transport Research Conference, September 7-11, Cambridge, UK.

Hensher, D.A., Ton, T.T., 2000. A comparison of the predictive potential of artificial neural networks and nested logit models for commuter mode choice. Transp. Res. E 36, 155-172.

Knowles, R.D., 1996. Transport impacts of Greater Manchester's Metrolink light rail system. J. Transp. Geography 4 (1), 1-14.

Mackett, R.L., Edwards, M., 1998. The impacts of new urban public transport systems: will the expectations be met? Transp. Res. A 32, 231-245.

Metro Development Study, 1996a. Public Transport Mode Inventory for Athens, Attiko Metro Agency, Athens, Greece.

Metro Development Study, 1996b. Private Transport Mode Inventory for Athens, Attiko Metro Agency, Athens, Greece.

Monzon, A., 2000. Travel demand impacts of a new privately operated suburban rail in the Madrid N-III corridor, Presented in the European Transport Research Conference, September 7-11, Cambridge, UK

Newman, P., 1995. Public transit: the key to better cities. Siemens Rev. $3-4,42-46$

Thornblom, R., Bengtsson, J.E., 1997. New Stockholm metro car - an underground pilot project. Public Transp. Int. 46 (3), 39-41, see also pp. 44-45.

Vuchie, V.R., 1991. Recognizing the value of rail transit. TR News $156,13-19$

Washington, S., Karlaftis, M.G., Mannering, F.L., 2002. Statistical and Econometric Methods for Transportation Data Analysis. Chapman \& Hall/CRC Press, Boca Raton, FL (forthcoming). 